\title{
Formation of Ligand Clusters on Multimodal
}

\section{Chromatographic Surfaces}

Camille L.Bilodeau ${ }^{+}($bilodc2@rpi.edu), Edmond Y. Lau(lau12@IInl.gov), David Roush*

(david_roush@merck.com), ShekharGarde (gardes@rpi.edu), Steven M.

$\operatorname{Cramer}^{\dagger}$ (crames@rpi.edu)

${ }^{\dagger}$ Howard P. Isermann Department of Chemical and Biological Engineering and Center for

Biotechnology and Interdisciplinary Studies, Rensselaer Polytechnic Institute, 110 Eighth Street,

Troy, New York 12180, United States of America

${ }^{\ddagger}$ Physical and Life Sciences Directorate, Lawrence Livermore National Laboratory, Livermore,

California, 94550, United States of America

*Biologics and Vaccines, Downstream Process Development and Engineering, Merck \& Co., Inc., Kenilworth, NJ, 07033, United States of America

\section{Supporting Information Text} Time Dependence of Moiety Density Distribution

In this manuscript, we report the moiety density distribution for a variety of ligand systems averaged over 45 ns (with $5 \mathrm{~ns}$ removed from the beginning of the trajectory for equilibration). To illustrate how these systems change as a function of time, Figure S1 shows these moiety density distributions averaged over two 5 ns parts of the trajectory: 5-10 ns and 45-50 ns. 

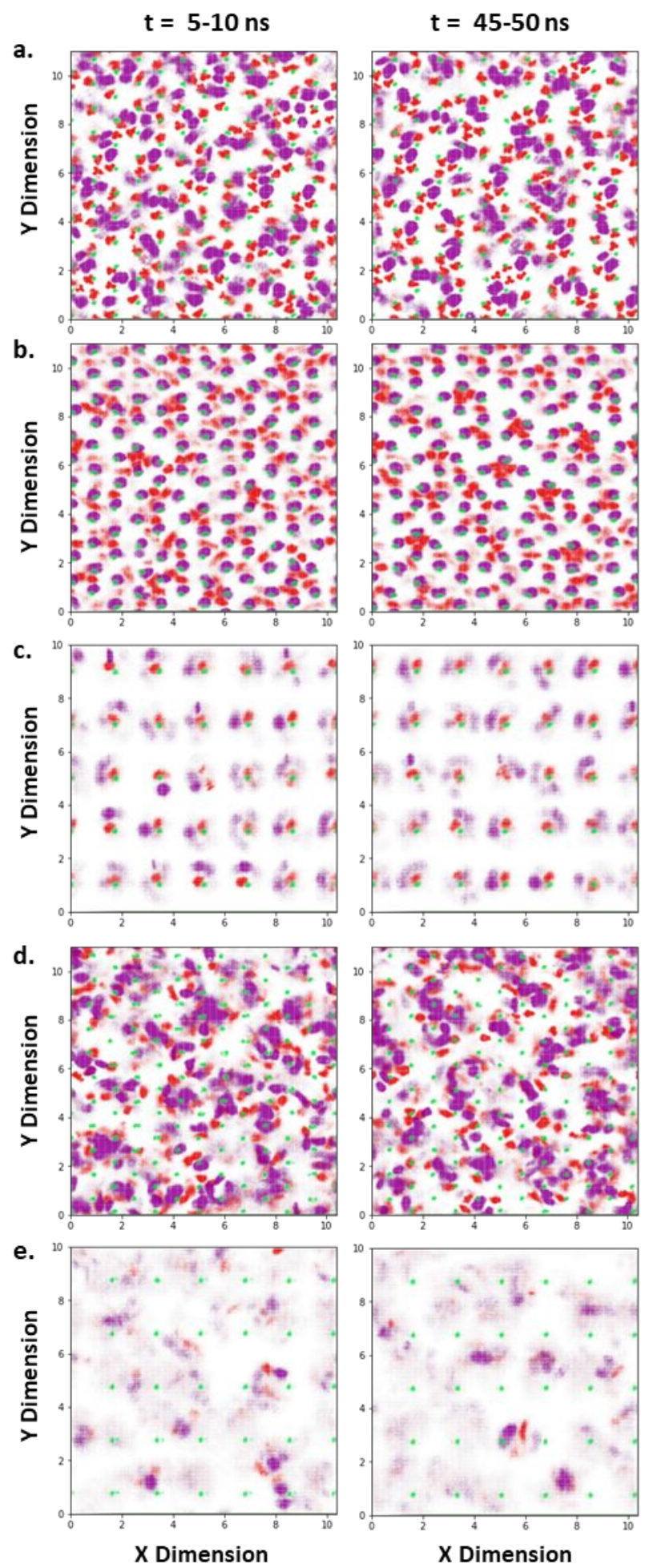

Figure S1. Density distributions for (a) the Capto Ligand, (b) Nuvia cPrime, (c) the Capto Ligand at low ligand density, (d) the Capto Ligand with linker, and (e) the Capto Ligand with linker at low ligand density immobilized on a SAM surface. Distributions shown on the left are taken from $5 \mathrm{~ns}$ to $10 \mathrm{~ns}$ (keeping the first $5 \mathrm{~ns}$ for equilibration). 
Distributions on the right are taken from $45 \mathrm{~ns}$ to $50 \mathrm{~ns}$. Atom density scale ranges from 0 (white) to 2 atoms $/ \mathrm{nm}^{2}$ (relevant color). Colors: Purple, phenyl ring; red, carboxylic acid group; green, base atom.

\section{Moiety Density Distribution for Additional Simulations}

In the manuscript, we illustrated the moiety density distribution for one 50 ns simulation. To illustrate that the qualitative nature of the surface does not change for replicate simulations, Figure S2 shows surfaces generated with different starting velocities. 

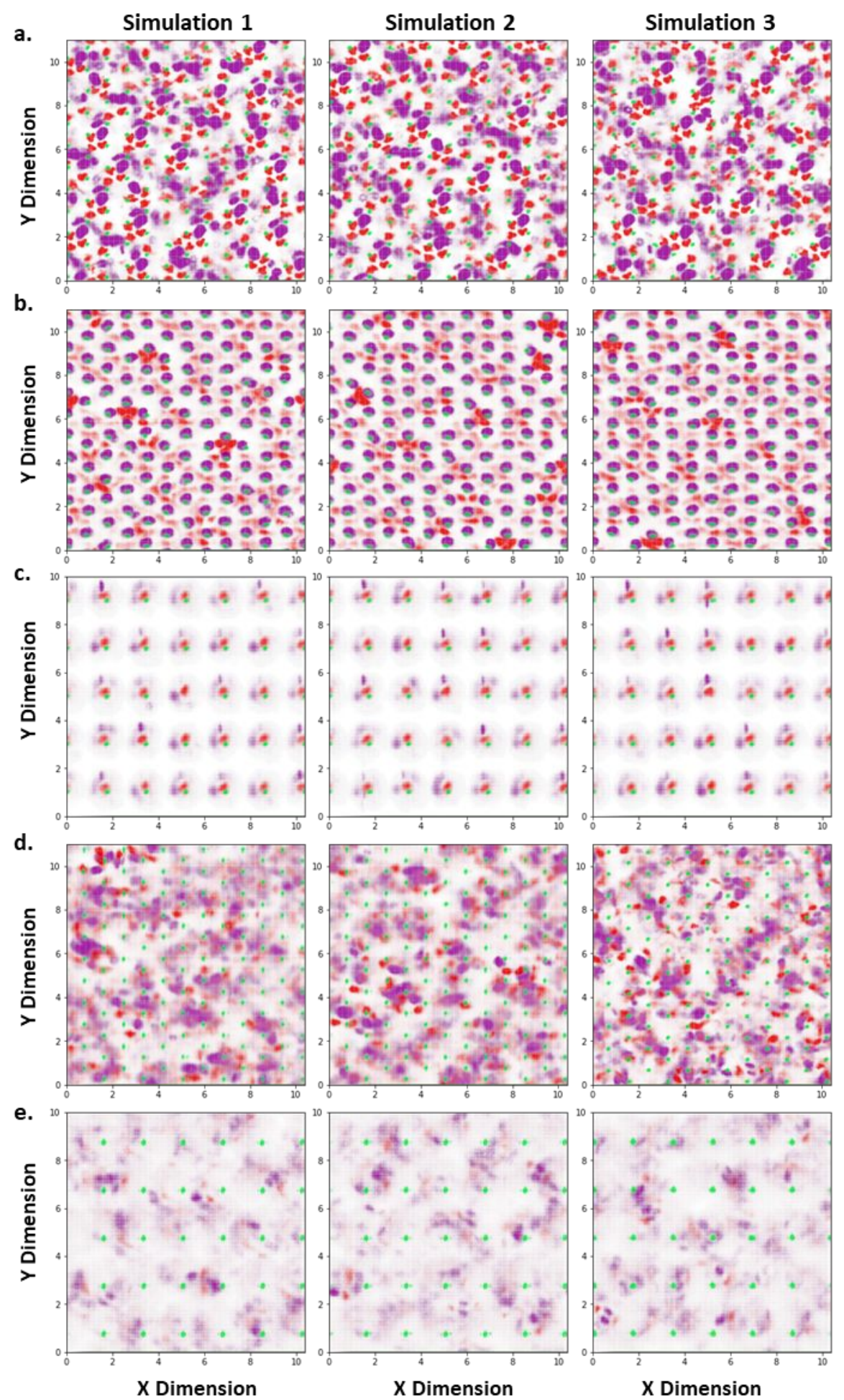

Figure S2. Density distributions for (a) the Capto Ligand, (b) Nuvia cPrime, (c) the Capto Ligand at low ligand density, (d) the Capto Ligand with linker, and (e) the Capto Ligand with linker at low ligand density immobilized on a SAM surface. Left, middle, and right distributions are taken from three different simulations with initial velocities 
generated from different seed values. Atom density scale ranges from 0 (white) to 2 atoms $/ \mathrm{nm}^{2}$ (relevant color). Colors: Purple, phenyl ring; red, carboxylic acid group; green, base atom. 